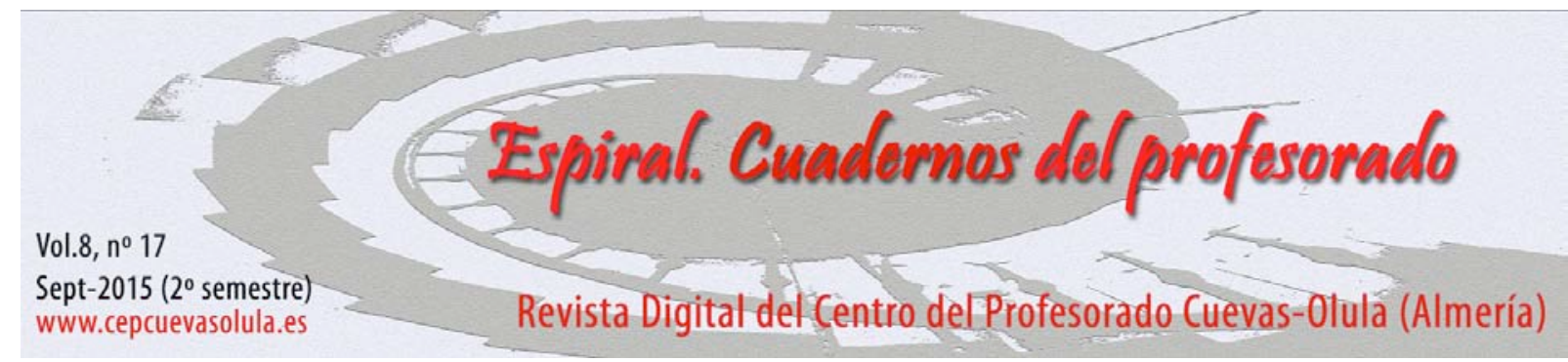

\title{
LAS FINALIDADES DEL DIÁLOGO EN LOS GRUPOS INTERACTIVOS
}

\author{
The purposes of the dialogue in interactive groups
}

\section{Estefanía Fernández Antón}

Doctoranda Investigación e Innovación en Educación, Universidad de Segovia

\begin{abstract}
RESUMEN: Los grupos interactivos son una actuación socioeducativa de éxito en la cual los grupos heterogéneos de alumnos y la interacción dialógica entre estudiantes, profesorado, familiares y otras personas de la comunidad son fundamentales para el proceso de enseñanza y aprendizaje. Teniendo en cuenta estas ideas nos planteamos como objetivo dar a conocer las finalidades del diálogo en los grupos interactivos de una Comunidad de Aprendizaje. Para alcanzar la finalidad expuesta, empleamos la metodología comunicativa crítica y las técnicas de recogida de datos la observación comunicativa y la entrevista en profundidad. Desde el procedimiento metodológico anterior, conocemos que el diálogo es necesario para el avance individual y para el avance del grupo de niños que conforman la clase. Esto significa que los niños y niñas no aprenden individualmente, sino que todos aprenden juntos y los aprendizajes individuales son fundamentales para favorecer el aprendizaje de las otras personas.
\end{abstract}

Palabras clave: aprendizaje en grupo, diálogo, relación escuela-comunidad.

ABSTRACT: The interactive groups are an actuation of successful in which the heterogeneous groups of students and the dialogic interaction between students, professorate, family and other persons of community are necessary for the development of process de teaching and learning. Taking into account these ideas, we propose like objective give to know the purposes of dialogue in the interactive groups of a Learning Communities. We use the critic communicative methodology and the techniques for collect information the communicative observation and the interview in deep for achieves the finality previous. From the above methodological procedure, we know that dialogue is necessary for the advance individual and for the advance of student of the classroom. This means that children do not learn individually, they learn together. Individual learning is essential to encourage the learning of others.

Key words: aprendizaje en grupo, diálogo, relación escuela-comunidad.

Fernández Antón, E. (2015). Las finalidades del diálogo en los grupos interactivos. Espiral. Cuadernos del Profesorado, 8(17), 17-34. Disponible en: http://www.cepcuevasolula.es/espiral.

Fecha de recepción: 26/05/2015

Fecha de aceptación: 17/07/2015
Enviar correspondencia a: fanitafernandez@hotmail.com 


\section{1.- INTRODUCCIÓN}

De la conciliación de Freire en la educación, de Bruner y Vigotsky en la psicología y en el enfoque socio histórico del desarrollo y de Habermas en la sociología (entre otros) CREA (Centro Especial de Investigación en Teorías y Prácticas Superadoras de Desigualdades) desarrolla el aprendizaje dialógico que contiene diferentes principios dialógicos. Estos son necesarios para que las personas de la comunidad (familias, docentes, alumnado y otras personas del entorno) aprendan a tener en cuenta las opiniones de todas las personas, académicas y no académicas. También permiten adquirir habilidades académicas, comunicativas y sociales, que son útiles para la vida cotidiana. Todos estos objetivos se consiguen desde el diálogo igualitario que explicaremos a continuación (Aubert, Duque, Fisas, y Valls, 2004).

Según Aubert et al. (2004) "si tuviéramos que definir el aprendizaje dialógico de la forma más sencilla, diríamos que es aquel aprendizaje que resulta del diálogo igualitario, esto es, del diálogo entre iguales basado en pretensiones de validez" (p. 124). Habermas (1999) nos aclara el significado de ambas pretensiones: las primeras son permisibles con la afirmación o la negación razonada y con el entendimiento para llegar a consensos. En los actos comunicativos de poder, las expresiones de rechazo o aceptación son arbitrarias dando lugar a situaciones de desigualdad.

Siguiendo con el aprendizaje dialógico, hemos de decir que es el marco teórico del proyecto educativo y social Comunidades de Aprendizaje (Valls y Munté, 2010) y de actuación educativas de éxito grupos interactivos (Adell, Herrero, y Siles, 2004). Aquí hablaremos de ambos temas, pues nuestro objetivo es dar a conocer las finalidades del diálogo en los grupos interactivos de una Comunidad de Aprendizaje.

Teniendo en cuenta las ideas establecidas en el párrafo previo, ahora nos toca indicar que una Comunidad de Aprendizaje es definida por Jaussi y Luna (2002) de la siguiente manera:

"Las Comunidades de Aprendizaje son un proyecto de transformación de los centros educativos para responder a los nuevos retos educativos. Parte del análisis de la actual sociedad, de las experiencias de éxito que en distintas partes del mundo están dando respuesta a esas necesidades y problemas, y de las teorías sociales más referenciadas actualmente, las de orientación comunicativa y reflexiva (pp. 40-41)".

En la definición anterior, cuando se habla del análisis de la sociedad actual se está haciendo referencia a la sociedad de la información. De acuerdo con Valls (2008), las Comunidades de Aprendizaje en relación a la sociedad de la información son “... una respuesta educativa igualitaria [...] para todos y todas, en las que se parte de que todos los niños y niñas tienen derecho a la mejor educación, tienen capacidades para conseguirla y de que nadie está condenado de antemano a tener una posición educativa y cultural marginal" (p. 222).

Las Comunidades de Aprendizaje ponen en práctica diferentes actuaciones socioeducativas de éxito como los grupos interactivos, aunque éstas son transferibles a otros contextos (Flecha, García, y Gómez, 2013; Ojala y Padrós, 2012).

Los Grupos Interactivos son una organización formada por grupos heterogéneos de alumnos que tiene como objetivo la interacción dialógica entre estudiantes, profesorado, familiares y otras personas de la comunidad. Esta interacción el aprendizaje de los contenidos necesarios para formar parte de la sociedad de la información (Molina, 2011).

En cuanto a su organización, los Grupos Interactivos están constituidos por pequeños grupos heterogéneos de cinco o seis estudiantes. Cada grupo es coordinado por un voluntario o voluntaria, y todos ellos son supervisados por el responsable de aula, que es un docente del centro (Aubert y García, 2001). Los docentes también tienen que evaluar el funcionamiento de los grupos interactivos con la ayuda del voluntariado (Odina, Buitago, y Alcalde, 2006, p. 98).

En el caso de la Comunidad de Aprendizaje de este trabajo la evaluación se realizaba durante el desarrollo y al final de los grupos interactivos. En el primer momento, el responsable de aula recogía datos sobre el desarrollo del alumnado fuera de cada agrupación, y las personas voluntarias rellenaban una ficha elaborada por el docente. En esta ficha se tenía en cuenta las habilidades 
académicas, comunicativas y sociales del alumnado. En el segundo momento el voluntariado, los docentes y el alumnado se reunían para dialogar sobre los acontecimientos y las capacidades que tenía el alumnado y las que tenía que transformar.

En último lugar, queremos indicar que aparte de este trabajo, hay muchos otros que están relacionados con los grupos interactivos: Elboj y Niemelä (2010), Oliver y Gatt (2010), Molina (2011), Racionero (2010) y Valls y Kyriakides (2013). En ellos se demuestra que esta actuación educativa de éxito está pensada para que todos los alumnos alcancen el éxito académico.

\section{2.- METODOLOGÍA}

En un contexto como las Comunidades de Aprendizaje, el proceso de enseñanza y aprendizaje está sustentado en el diálogo y en la participación de la comunidad; así pues la metodología comunicativa crítica puede ser apropiada para obtener datos por los motivos expuestos a continuación.

Desde esta metodología, hay que tener en cuenta las voces de los participantes en relación a la interpretación de los hechos; esto se puede conseguir a través del diálogo. También se puede transformar la realidad en beneficio de todos y todas (Gómez y Díez, 2009).

Teniendo presente las anteriores ideas, queremos decir que nosotros elegimos un procedimiento metodológico comunicativo crítico con orientación cualitativa. Los procedimientos que llevamos a cabo fueron los expuestos en el párrafo anterior, las técnicas de investigación fueron la observación y la entrevista y; por último, las herramientas elegidas fueron el cuaderno, el diario y la grabadora.

Las observaciones tuvieron una orientación comunicativa porque seguimos el siguiente proceso: compartimos los objetivos del estudio antes de la primera observación, recogimos los hechos ocurridos, interpretamos la realidad con la ayuda de los participantes y dialogamos sobre los acontecimientos al final de cada sesión de observación (Gómez, 2012). Las observaciones se recogieron el 14.12.12; el 1.2.13; el 15.2.13; el 22.2.13; el 8.3.13; el 15.3.13 y el 22.3.13. En ellas participaron voluntarios, docentes y alumnos.

Las entrevistas fueron en profundidad, y sus participantes fueron dos madres que eran personas voluntarias del centro de manera sistemática. Las entrevistas tuvieron lugar el 31.1.14 y el 7.3.14.

Antes de pasar a las herramientas, vamos a detallar el código utilizado para proteger la identidad de los participantes de las observaciones y el número total de personas que formaron parte de este trabajo en la siguiente tabla. Sobre el código también indicar que nos sirve para conocer sobre qué persona estamos hablando en los resultados.

Tabla 1. Participantes del estudio. Fuente: elaboración propia

\begin{tabular}{|c|c|}
\hline \multicolumn{2}{|l|}{ Participantes } \\
\hline \multicolumn{2}{|l|}{ Docentes } \\
\hline \multicolumn{2}{|l|}{ «A»y «AB». } \\
\hline \multicolumn{2}{|l|}{ Voluntariado } \\
\hline Personas voluntarias & «S» y la autora de este trabajo. \\
\hline \multicolumn{2}{|l|}{ Alumnado } \\
\hline $\begin{array}{l}\text { Alumnos que ya no están en la } \\
\text { escuela }\end{array}$ & «An», «C»y $\langle\mathrm{E} »$. \\
\hline Alumnado de cuarto de Primaria & $« \mathrm{Cr} »,\langle\mathrm{Di} » \mathrm{y} \ll \mathrm{V} »$ \\
\hline Alumnado de quinto de Primaria & «DN»y «Ne». \\
\hline Alumnado de sexto de Primaria & $\langle\mathrm{M} » \mathrm{y}\langle\mathrm{P} »$ \\
\hline Total & 14. \\
\hline
\end{tabular}


Como ya hemos indicado más arriba, las herramientas que nos ayudaron a recoger los datos son el cuaderno, el diario y la grabadora. En el cuaderno apuntábamos los sucesos en general y las acciones de los participantes en los grupos interactivos de manera más específica.

En el diario anotábamos nuestras reflexiones y opiniones en función de la información recogida en las observaciones y en las entrevistas. La información de esta última técnica de investigación se recogía con la grabadora, se transcribía y, como ya hemos dicho, se acompañaba de reflexiones y opiniones propias.

\section{3.- RESULTADOS}

En las próximas líneas daremos a conocer las finalidades del diálogo en los grupos interactivos de una Comunidad de Aprendizaje.

Dentro de un proceso de enseñanza y aprendizaje basado en el aprendizaje dialógico, el alumnado tenía que compartir los conocimientos académicos adquiridos. Así pues, el saber aislado o particular se convirtió en el origen del saber comunitario, que fue desarrollado teniendo en cuenta el conocimiento de todos los niños:

... «A $\mathrm{A}$ me muestra cómo hay que incitar a los escolares para que se ayuden entre ellos—: Pensar de qué trata la actividad. «V», el alumno más pequeño, no entiende en qué consiste, por eso, «A» dice-: Algún compañero que lo entienda que lo explique al resto.

«An» ayuda a « $\mathrm{V} » —$ : Tienes que hacer el doble del número y poner la palabra en el resultado correcto.

«V» le pregunta a «An»—: ¿Entonces, tengo que poner la palabra en estos huecos?

«An» le contesta—: Sí, porque así se forma la frase (Observación comunicativa: 14.12.12).

De lo que estamos hablando en el anterior ejemplo es de la importancia no solo del aprendizaje individual, sino también del aprendizaje colectivo; en otras palabras, la persona tiene su proceso de aprendizaje y sus conocimientos propios y, desde estos, todos aprenden de todos.

Esta forma de aprendizaje fue positiva para los escolares que no entendieron las tareas y, como vemos en el anterior ejemplo y en el posterior, fue desarrollada desde el diálogo entre los estudiantes:

Después de leerlo dos veces, «Ne»y «M» reconocen no entenderlo. «Cr» tampoco lo entiende muy bien, aun así intenta explicárselo a sus dos compañeras-: Hay que poner un número a cada letra y hacer esta suma (señala el sentido de los números horizontales).

— ¿No se os olvida algo? — les pregunto.

Los cuatro me miran mientras discurren que les falta. De pronto «An» dice entusiasmado-: También es la suma así (señala el sentido de los números verticales)... (Observación comunicativa: 1.2.13).

El diálogo tenía lugar desde las preguntas y contestaciones del alumnado. Las personas voluntarias aprovechábamos estas conversaciones de los estudiantes para solicitar que las respuestas fuesen más apropiadas y detalladas. Por lo tanto, era una forma de repasar contenidos académicos y de asegurar que los conocimientos adquiridos fuesen rigurosos:

... «Cr» rompe el silencio con una pregunta-: ¿Qué es un hiato, «An»? —Dos vocales en una misma sílaba - contesta «An».

«Cr» sonríe al ver a su compañero tan seguro y añade-: Eso es el diptongo.

—Vale, pues entonces cuando las vocales están en silabas distintas — dice «An».

«AB» está escuchando la respuesta de «An», por eso, añade—: Antes de dar una contestación, tienes que pensar mucho. (Observación comunicativa: 8.3.13). 
... «V» empieza a preguntar a «C»—: ¿Qué es una palabra compuesta? —Sacapuntas - dice $« \mathrm{C} »$.

Entonces, decido animar a «C» para que dé una respuesta más detallada—: ¿Qué te parece intentar aclarárselo con más detalle?

«C» se incorpora un poco de la silla y le explica a «V»—: Es una palabra con varias palabras juntas. (Observación comunicativa: 8.3.13).

Los escolares también dialogaron para llegar acuerdos sobre la resolución de los ejercicios académicos. Esto permitía que todos participaran dando sus opiniones y se sintieran escuchados por el resto de compañeros que formaban el grupo:

Posteriormente los niños y niñas de la mesa tres empiezan a crear cuentas con cuatro cuatros. «An» propone la cuenta $4+4+4+4=$. - $i$ Os parece bien esta cuenta? — pregunta «An» a todos los componentes de la mesa.

—Creo que es muy fácil — dice «M» (alumna de quinto de Primaria).

- Poner otra si queréis — dice «An». — Venga «Di», pon otra cuenta.

- Vale, podemos sumar tres cuatros y que se reste uno — dice «Di». A todos los niños les parece bien la idea de «Di» (Observación comunicativa: 15.3.13).

Como vemos en el ejemplo anterior, todas las opiniones eran diferentes y todas ellas eran bien acogidas. No importaba quien aportaba la idea, sino el valor de la misma. Esta afirmación está relacionada con el diálogo igualitario, que es uno de los principios del aprendizaje dialógico (Flecha, 2011).

En cuanto a las personas voluntarias, tenemos que señalar que nuestro papel como persona adulta no era transmitir conocimientos o solucionar las actividades, nuestra función era orientar al alumnado hacia la solución correcta mediante el diálogo. En la orientación solo se podía promover la reflexión de los estudiantes a través de preguntas y, en estos interrogantes, no podía hacerse referencia a las soluciones:

«E» sigue ayudando a «P» a corregir los errores de ortografía que hay en el ejercicio, pero en uno de ellos aprecio como «E» está diciéndole algo mal a «P»—: Muy bien, has dejado la tilde de la palabra él (la frase es él Rey...). — ¿Estás seguro «E»? —le pregunto.

—Sí, lleva tilde porque es diptongo — dice «V» sin dejar contestar a «E».

—Los diptongos son otra cosa —añade «C»... (Observación comunicativa: 22.2.13).

Como hemos indicado al principio de este trabajo, en la Comunidad de Aprendizaje de este estudio, había un momento de evaluación que tenía lugar después de los grupos interactivos. En este momento, todos los participantes de los grupos interactivos indicábamos las transformaciones académicas y sociales del alumnado y las que tenían que alcanzar. Esta información se transmitía a través del diálogo:

—Escuchad todos — dice «A», antes de terminar la sesión-. «M» ha cambiado mucho y «V» también, aunque todavía le falta poner más interés. «Ne» está cambiando poco a poco. «An», a mí me sorprendes porque dejas actuar a los demás y reflexionas sobre las correcciones para ponerlas en práctica. A «E» todavía le sienta mal cuando le comentamos un cambio que tiene que realizar.

-Es verdad, me cuesta mucho, pero lo estoy intentado — dice «E». (Observación comunicativa: 15.3 .13$)$.

... « $\mathrm{AB} » \mathrm{y}$ « $\mathrm{S}$ » piensan que « $\mathrm{M} » \mathrm{y}$ «V» han mostrado más interés que otros días. Por otro lado, yo digo que en mi mesa han estado un poco distraídos. «A» apunta las dos opiniones y se dirige a los dos niños-: Es verdad que hoy os habéis implicado mucho más, sin embargo, me gustaría que «V» estuviera un poco más atento en las actividades. 
«DN» da la razón a «A»-: «V» ha participado más, pero a veces no estaba muy atento. (Observación comunicativa: 15.2.13).

En estos diálogos, siempre que se hablaba de transformaciones alcanzadas, hacíamos referencias a los conocimientos, habilidades o actitudes que el alumnado estaba demostrando en los grupos interactivos y en los grupos interactivos en relación a diferentes situaciones vividas en la escuela o fuera de ella.

Asimismo, cuando se hacía referencia a las transformaciones que se tenían que alcanzar, estábamos hablando de los conocimientos, habilidades o actitudes que el alumnado tenía que lograr en la actuación socioeducativa de éxito y en esta actuación en relación a diferentes situaciones vividas en la escuela o fuera de ella.

Algunos ejemplos de los aprendizajes para la escuela y fuera de ella que permitían los grupos interactivos aparecen a continuación:

Yo creo que sobre todo son los conocimientos actitudinales, de saber comportase y de saber trabajar en grupo. Ahora mismo, en cualquier trabajo o en la universidad, trabajar en grupo es esencial. Ellos van a saber manejarse en un grupo, a interactuar con otras personas y a no tener miedo a dar su opinión. Yo creo que van a ser niños que van a cuestionar lo que les digan y van a dar su opinión. Más allá de mejorar los conocimientos instrumentales, creo que los Grupos ayudan mucho a mejorar la actitud y las relaciones con los demás (Entrevista: 31.1.14).

... les sirve para la vida, así buscan una cosa que no entienden, se sacan las castañas del fuego y salen adelante. Yo creo que lo van a pensar más, y se van a lanzar más a hacer las cosas. Yo creo que les viene muy bien porque se atreven más. (Entrevista: 7.3.14).

Debido a la naturaleza de la observación comunicativa, decidimos fortalecer nuestras interpretaciones con las ideas de las personas de la comunidad. En una conversación con una docente del centro, nos dimos cuenta de que la transformación del alumnado era un objetivo que tenía programado el profesorado del centro porque tenía ventajas para el desarrollo de los estudiantes:

- He descubierto que, en la valoración final, los adultos os preocupáis por compartir y comunicar los futuros cambios que tiene que hacer el alumnado, ¿crees que tengo razón? - le pregunto a «A».

- Creo que es una forma de beneficiar a los alumnos, pues ellos ven cómo van cambiando y cómo tienen que ir avanzando - dice «A». (Observación comunicativa: 22.3.13).

Finalmente, queremos indicar que en los diálogos entre todos los participantes de la comunidad, las personas adultas queríamos tener en cuenta las opiniones de los estudiantes, por eso, cuando no eran detalladas les dimos la opción de ampliarlas:

La valoración de los alumnos está llegando al final, sólo falta la intervención de «M»-: «Di» ha estado regular. Estas palabras no satisfacen a «AB»—: ¿Qué significa regular?

«M» permanece callada, por eso, «AB» añade-: En todo caso hay que decir porqué un compañero lo ha hecho bien o mal.

- Porque no ha participado mucho - manifiesta «M». (Observación comunicativa: 22.2.13).

En el semicírculo que forman los alumnos y alumnas, el grupo uno es el primero en intervenir. «M» tiene que valorar las acciones de «Cr», pero parece que a «M» la domina la timidez y sólo dice- : «Cr» lo ha hecho bien.

« $\mathrm{AB}$ » la dice- : Hay que intentar pensar un poco, es decir, tienes que decir porqué un compañero lo ha hecho bien o mal. Sin embargo, «M» tiene el cuerpo encogido por la timidez y no aporta nada (Observación comunicativa: 8.3.13). 
Los anteriores ejemplos es una forma de demostrar que las opiniones de los estudiantes eran tan importantes como las de un adulto.

\section{4.- DISCUSIÓN Y CONCLUSIONES}

En los grupos interactivos las interacciones dialógicas entre los niños y los adultos; estos últimos representados por los docentes, familiares y personas de la comunidad, son fundamentales para alcanzar diferentes finalidades relacionadas con el proceso de enseñanza y aprendizaje (Molina, 2011).

Desde las interacciones que acabamos de explicar, el alumnado no es un individuo que aprende solo y tiene conocimientos para sí mismo; sino que todos aprenden juntos y los aprendizajes individuales son la base para el aprendizaje de las otras personas. Esta forma de aprendizaje es de gran ayuda para los alumnos a los que les cuesta llevar acabo algunas tareas.

Así pues entendemos que los grupos interactivos ayudan al alumnado a darse cuenta de que no es un individuo que aprende solo y para sí mismo; es un individuo que tiene responsabilidad sobre el aprendizaje de los otros (Racionero, 2010).

El diálogo en los grupos interactivos tenía muchas otras finalidades necesarias para el desarrollo del alumnado. Todas ellas son necesarias para el avance individual y para el avance del grupo; los ejemplos de los que hemos hablado con anterioridad son: contestar a las dudas de los compañeros con respuestas profundas y cercanas a la realidad, dar ideas y sentir que éstas están siendo escuchadas, valorar todas las ideas (independientemente de la personas que realice la aportación), reflexionar sobre la respuesta y desarrollar o transformar conocimientos, habilidades y actitudes para la escuela y para la vida fuera de ella.

Entonces, desde las últimas ideas de desarrollo y transformación, vemos como los conocimientos, habilidades o actitudes que se adquieren en los grupos interactivos no están relacionadas sólo con la escuela, sino también con la sociedad en general. Es decir, se está conectando escuela y sociedad.

Este trabajo, como muchos otros relacionados con los grupos interactivos, nos parecen interesantes porque se centran en un tipo de aprendizaje basado en el diálogo y, por lo tanto, que está en consonancia con la sociedad en la que vivimos (Gómez y Díez, 2009).

\section{5.- REFERENCIAS}

Adell, M. J., Herrero, C. \& Siles, B. (2004). El aprendizaje dialógico en los Grupos Interactivos. An On-line Journal for Teacher Research, 7(1). Recuperado de (http://journals.library.wisc.edu/index.php/networks/article/view/30/35) [20 mayo 2015]

Aubert, A., Duque, E., Fisas, M. \& Valls, R. (2004). Dialogar y transformar. Pedagogía crítica del siglo XXI. Barcelona: Graó.

Aubert, A. \& García. C. (2001). Interactividad en el aula. Cuadernos de Pedagogía, 301, 20-24.

Elboj, C. \& Niemelä, R. (2010). Sub-communities of mutual learners in the classroom: the case of interactive groups. Revista de Psicodidáctica, 15(2), 177-189.

Flecha, R. (2011). Compartiendo palabras. El aprendizaje de las personas adultas a través del diálogo. Barcelona: Paidós.

Flecha, R., García, R., \& Gómez, A. (2013). Transferencia de Tertulias Literarias Dialógicas a instituciones penitenciarias. Revista de Educación, 360, 140-161.

Gómez, A. \& Díez, J. (2009). Metodología comunicativa crítica: transformaciones y cambios en el S.XXI. Revista Electrónica Teoría de la Educación. Educación y Cultura en la Sociedad de la Información, 10(3), 103-118.

Gómez, J. (2012). Metodología comunicativa crítica. En R. Bisquerra (Coord.), Metodología de la investigación educativa (pp. 395-424). Madrid: La Muralla.

Habermas, J. (1999). Teoría de la Acción Comunicativa. Volumen I: racionalidad de la acción y racionalización social. Madrid: Taurus. 
Jaussi, M. L. \& Luna, F. (2002). Comunidades de Aprendizaje. Transformar en lugar de adaptar. Cuadernos de Pedagogía, 316, 40-41.

Molina, S. (2011). Los Grupos Interactivos: una práctica de las Comunidades de Aprendizaje para la inclusión del alumnado con discapacidad (Tesis doctoral). Recuperada de Tesis Doctorales en Red (http://hdl.handle.net/2445/43112) [20 mayo 2015]

Odina, M., Buitago, M. \& Alcalde, A. I. (2006). Los Grupos Interactivos. En F. López (Dir.), Transformando la escuela: Comunidades de Aprendizaje (pp. 97-102). Barcelona: Graó.

Ojala, M. \& Padrós, M. (2012). Actuaciones de éxito, universales y transferibles. Cuadernos de Pedagogía, 429, 18-19.

Oliver, E. \& Gatt, S. (2010). De los actos comunicativos de poder a los actos comunicativos dialógicos en las aulas organizadas en Grupos Interactivos. Signos, 43(2), 279-294.

Racionero, S. (2010). Egalitarian dialogue and instrumental dimension. Two principles of dialogic learning in the classroom. Psychology, Society, \& Education, 2(1), 61-70.

Valls, R. (2008). Comunidades de Aprendizaje: una práctica educativa de aprendizaje dialógico para la sociedad de la información (Tesis doctoral). Recuperada de Tesis Doctorales en Red de (http://hdl.handle.net/10803/2929) [20 mayo 2015]

Valls, R. \& Kyriakides, L. (2013). The power of interactive groups: how diversity of adults volunteering in classroom groups can promote inclusion and success for children of vulnerable minority ethnic populations. Cambridge Journal of Education, 43, 17-33.

Valls, R. \& Munté, A. (2010). Las claves del aprendizaje dialógico en las Comunidades de Aprendizaje. Revista Interuniversitaria de Formación del Profesorado, 67(24, 1), 11-15. 